\title{
QUASI-CONTINUOUS FUNCTIONS ASSOCIATED WITH A HUNT PROCESS
}

\author{
YVES LE JAN
}

\begin{abstract}
A stochastic characterization is given for quasi-continuous functions associated with Hunt processes.
\end{abstract}

Introduction. Quasi-continuous functions have been studied in various frameworks of potential theory, especially Dirichlet spaces (cf. $[1,4,5]$ ). The main object of this paper is to establish the relation between the quasi-continuity and the quasi left continuity of processes. Under rather strong duality assumptions, it also appears that the quasi-continuous functions are the functions which are finely and cofinely continuous.

1. Definitions. Let $X_{t}$ be a Hunt process defined on a compact space $E$ containing a cemetery $\Delta$ with a reference measure $m$ we may assume to be bounded. In the sequel we shall use mostly the notations of [2], and all functions and sets will be implicitly assumed nearly Borel.

A bounded sequence of functions $f_{n}$ is said to be converging quasi-uniformly towards $f$ iff for some $\alpha>0$ there exists a sequence $g_{n}$ of $\alpha$-excessive functions decreasing to $0 \mathrm{~m}$ a.s. (and therefore quasi everywhere (cf. [2, Proposition 3.2, p. $280])$ ), such that $\left|f-f_{n}\right|$ is smaller than $g_{n}$.

This definition is independent of $\alpha$. Indeed, for any $\beta>0, g_{n}+(\alpha-\beta) U^{\beta} g_{n}$ is $\beta$ excessive, decreases to 0 q.e. and dominates $\left|f-f_{n}\right|$.

One can give an alternate and more intuitive definition of the quasi-uniform convergence.

Proposition 1. A bounded sequence $f_{n}$ converges quasi-uniformly iff there exists a sequence $G_{m}$ of open sets such that

(a) $f_{n}$ converges uniformly on every set $E-G_{m}$;

(b) the capacitary potentials $e_{G_{m}}^{\alpha}=E_{x}\left(e^{-\alpha T_{G_{m}}}\right)$ are decreasing to $0 \mathrm{~m}$ a.s. (and therefore quasi everywhere), for $\alpha>0$.

REMARK. The property (b) is independent of $\alpha$ and equivalent to

$\left(\mathrm{b}^{\prime}\right)$ the stopping times $T_{G_{m}}$ increase to $+\infty P_{\mu}$ a.s. for every initial distribution $\mu$ charging no polar set.

PROOF. The sufficiency is easy to check. If $f_{n}$ is uniformly bounded by $M$, for each $m,\left|f_{n}-f\right| \leq 2^{-m}+2 M e_{G_{m}}^{\alpha}$ for $n$ large enough, and the sequence of $\alpha$ excessive functions $2^{-m}+2 M e_{G_{m}}^{\alpha}$ decreases a.s. to 0 .

Received by the editors May 9, 1980 and, in revised form, November 18, 1980 and November $1,1981$.

1980 Mathematics Subject Classification. Primary 60J40; Secondary 60J45.

Key words and phrases. Hunt processes, capacity, quasi-continuity, quasi left continuity. 
To prove the converse, it is sufficient to construct a sequence of finely open sets $G_{m}$ (cf. [2, Theorem 11.2, p. 62]). Set $n_{j}=\inf \left(n, m\left(g_{n}\right) \leq 2^{-2 j}\right.$ ) and $F_{k}=$ $\bigcap_{j \geq k}\left\{g_{n_{j}} \leq 2^{-j}\right\}$. If $A_{j}=\left\{g_{n_{j}}>2^{-j}\right\}, 2^{j} g_{n_{j}} \geq 1_{A_{j}}$ and so $2^{j} g_{n_{j}} \geq e_{A_{j}}^{\alpha}$. Hence $m\left(e_{A_{j}}^{\alpha}\right) \leq 2^{+j} m\left(g_{n_{j}}\right) \leq 2^{-j}$. Thus, $m\left(e_{E-F_{k}}^{\alpha}\right) \leq \sum_{j \geq k} m\left(e_{A_{j}}^{\alpha}\right) \leq 2^{-(k-1)}$ and therefore $e_{E}^{\alpha}-F_{k}$ decreases to 0 q.e. as $k$ tends to $+\infty$. Clearly $f_{n}$ converges uniformly on each $F_{k}$.

We shall say that a bounded function $f$ is quasi-continuous iff it is a quasi-uniform limit of continuous functions. One checks easily that a quasi-uniform limit $f$ of quasi-continuous functions $f_{n}$ is quasi-continuous. (Find $f_{n}^{\prime}$ continuous and $h_{n} \alpha$ excessive such that $\left|f_{n}-f_{n}^{\prime}\right| \leq h_{n}$ and $m\left(h_{n}\right)<2^{-n} .\left|f-f_{n}^{\prime}\right| \leq \sum_{m \geq n} h_{m}+$ $\left|f-f_{n}\right|$ decreases to 0 quasi-uniformly.)

We shall say that a stochastic process is quasi-continuous iff it is right continuous and quasi left continuous (i.e. continuous on strictly increasing sequences of stopping times, i.e. such that its predictable projection is left continuous). In particular, if $f$ is a function on $E$, the process $f\left(X_{t}\right), t>0$, is quasi-continuous iff it is right continuous and the process $f\left(X_{t-}\right)$ (which is the predictable projection of $f\left(X_{t}\right)$ by [7]) is left continuous.

2. Stochastic characterisation of quasi-continuous functions. The main purpose of this paper is to prove the following:

THEOREM 1. For a bounded function $f$, the following properties are equivalent:

(A) $f$ is quasi-continuous,

(B) $f\left(X_{t}\right)$ is a.s. quasi-continuous on $(0+\infty)$,

(C) $\alpha U_{\alpha} f$ converges towards $f$ quasi-uniformly as $\alpha \uparrow+\infty$.

REMARK. The assumption that $m$ is a reference measure can be removed if the initial distributions are restricted to those which do not charge $m$-polar sets. This applies in particular to the case of processes associated to Dirichlet spaces, where the probabilities $P_{\mu}$ are canonically defined only for such initial measures (cf. [6, 8]).

Equivalence of $C$ and $B$.

(a) Necessity. It is well known that $U_{\alpha} f\left(X_{t}\right)$ is quasi-continuous. We only have to prove that the stochastic quasi-continuity is conserved in a quasi-uniform limit. With the notations of the definition, if $T_{m}$ is a sequence of stopping time converging towards a stopping time $T>0$, we have to prove that $f\left(X_{T_{m}}\right)-f\left(X_{T}\right)$ converges to 0 a.s.

By truncation, we can suppose that the stopping times are in some compact interval of $(0+\infty)$. Then it is clear that $Z_{M}=\sup _{m \geq M}\left|f\left(X_{T_{m}}\right)-f\left(X_{T}\right)\right|$ is, for all $n$, dominated by

$$
\begin{aligned}
\sup _{m \geq M} \mid f_{n}\left(X_{T_{m}}\right)- & f_{n}\left(X_{T}\right) \mid+g_{n}\left(X_{T}\right)+\sup _{m \geq M} g_{n}\left(X_{T_{m}}\right) \\
E\left(Z_{M}>\epsilon\right) \leq & E\left(\sup _{m \geq M}\left|f_{n}\left(X_{T_{m}}\right)-f_{n}\left(X_{T}\right)\right|>\epsilon\right) \\
& +E\left(g_{n}\left(X_{T}\right)+\sup _{m \geq M} g_{n}\left(X_{T_{m}}\right)>\epsilon\right)
\end{aligned}
$$


The second term can be made arbitrarily small by choosing $n$ large enough, using the maximal inequality for the supermartingale $e^{-\alpha t} g_{n}\left(X_{t}\right)$. Then the second term can be made arbitrarily small by choosing $M$, using the quasi-continuity of $f_{n}\left(X_{t}\right)$.

(b) Sufficiency. (i) Assuming $f\left(X_{t}\right), t>0$, stochastically quasi-continuous, let us show that $\alpha U_{\alpha} f \rightarrow f$ quasi-uniformly. We shall in fact prove that $P_{t} f \rightarrow f$ quasi-uniformly.

$$
\begin{aligned}
\sup _{u \leq t}\left|f(x)-P_{u} f(x)\right| & \leq \sup _{u \leq t} E_{x}\left(\left|f\left(X_{0}\right)-f\left(X_{u}\right)\right|\right) \\
& \leq E_{x}\left(\sup _{u \leq t}\left|f\left(X_{u}\right)-f\left(X_{0}\right)\right|\right) .
\end{aligned}
$$

We shall denote this latter function by $\phi_{t}(x)$. We have to prove that $\phi_{t}(x) \downarrow 0$ quasi-uniformly as $t \downarrow 0$.

(ii) Let us show that $\phi_{t}\left(X_{s}\right)$ is quasi-continuous $P_{\mu}$ a.s. for any initial distribution $\mu$. As a first step, let us prove that the process

$$
A_{s}^{t}=\sup _{s \leq u \leq t+s}\left|f\left(X_{u}\right)-f\left(X_{s}\right)\right|
$$

is quasi-continuous in $s$. If $T$ and $S$ are two stopping times, with $T<S$ on $\{S<$ $\infty\}$, one easily checks that

$$
\left|A_{T}^{t}-A_{s}^{t}\right| \leq \inf \left(\sup _{T \leq u \leq S}\left|f\left(X_{u}\right)-f\left(X_{T}\right)\right|, \sup _{T+t \leq u \leq S+t}\left|f\left(X_{u}\right)-f\left(X_{T+t}\right)\right|\right) .
$$

Therefore, since $f\left(X_{t}\right)$ is quasi-continuous, $\left|A_{T}^{t}-A_{s}^{t}\right|$ converges to zero as $T \uparrow S$ or as $S \downarrow T$.

(iii) Let us prove the quasi-continuity of $\phi_{t}\left(X_{s}\right)$ on $(0, u)$. For a sequence $T_{n}$ of stopping times increasing or decreasing towards $T$,

$$
\lim _{n \rightarrow \infty}\left(\phi_{t}(X)_{T_{n}}=\lim E\left(A_{T_{n}}^{t}\right)=E\left(A_{T}^{t}\right)=E\left(\phi_{t}(X)_{T}\right)\right.
$$

By Theorem 28 of Chapter IV of [3], this proves the right continuity and the existence of left limits. If $T$ is predictable, $E\left(\phi_{t}(X)_{T-}\right)=E\left(\phi_{t}(X)_{T}\right)$. For any $A \in \mathcal{F}_{T}, T_{A}$ is also predictable (since $\mathcal{F}_{T-}=\mathcal{F}_{T}$ ). If $S_{n}$ is an announcing sequence for $T_{A}$ and $S_{n}^{\prime}=S_{n} \wedge u, \lim _{n \rightarrow \infty} \Phi_{t}(X)_{S_{n}^{\prime}}=\Phi_{t}(X)_{T-1} 1_{A}+\Phi_{t}\left(X_{u}\right) 1_{A^{c}}$. Therefore applying the result to $T_{A} \wedge u$, we get that $E\left(\Phi_{t}(X)_{T-1}\right)=E\left(\Phi_{t}(X)_{T} 1_{A}\right)$, which allows us to conclude:

(iv) As $t \downarrow 0, \phi_{t}(x) \downarrow 0$ quasi everywhere.

We can now complete the proof with the following "Dini lemma":

LEMMA. If a bounded sequence $f_{n}$ decreases to 0 quasi everywhere, and if each process $f_{n}\left(X_{t}\right)$ is quasi-continuous, then the convergence is quasi-uniform.

Set $F_{n}=\left\{f_{n} \geq 2^{-k}\right\}$. It is enough to prove that $\lim _{n \rightarrow \infty} m\left(e_{\alpha}^{F_{n}}\right)=0$ for some $\alpha>0$. Indeed, if $m\left(e_{\alpha}^{F_{N(k)}}\right)<2^{-k}, f_{N(k)} \leq 2^{-k}+e_{\alpha}^{F_{N(k)}}$ and a fortiori, $f_{N(k)} \leq g_{k}$, with $g_{k}=\sum_{k}^{\infty}\left(e_{\alpha}^{F_{N(p)}}+2^{-p}\right)$. But $g_{k}$ is a sequence of $\alpha$ excessive functions decreasing to 0 a.s. since $m\left(g_{k}\right) \leq 2^{-k+2}$. Define $T_{n}=T_{F_{n}} ;$ since $f\left(X_{t}\right)$ is right continuous and $f_{n}$ decreasing, clearly $f_{n}\left(X_{T_{p}}\right) \geq 2^{-k}$ almost surely on $\left\{T_{p}<\infty\right\}$ for all $p \geq n$. Set $T=\lim _{n \rightarrow \infty} \uparrow T_{n}$. By the quasi left continuity, it is 
clear that $f_{n}\left(X_{T}\right) \geq 2^{-k}$ a.s. on $\{T<\infty\}$, for all $n$. Therefore $\lim _{n \rightarrow \infty} T_{n}=+\infty$ almost surely. It follows that $m\left(e_{F_{n}}^{\alpha}\right)=\lim \downarrow E_{m}\left(e^{-\alpha T_{n}}\right)=0$.

REMARK. It is easy to verify on the second part of the proof that a function $f$ is quasi-continuous as soon as $f\left(X_{t}\right)$ is quasi-continuous on $(0+\infty) P_{m}$ a.s.

Equivalence of $A$ and $B$. The stochastic quasi-continuity of continuous functions is obvious (since $X_{t}$ is quasi left continuous) and it has been observed that this property is stable under quasi-uniform limit.

Let $B$ be the Banach space obtained by completion of the space of stochastic quasi-continuous functions with respect to the norm $\|f\|=\inf (m(g), g$ 1-excessive, $g \geq|f|) . B$ is contained in $L^{1}(m)$ and the quasi-uniform convergence in $E$ implies the convergence in norm.

Since $|f| \leq|g|$ implies $\|f\| \leq\|g\|$, any continuous linear form on $B$ is the difference of two positive linear forms. By Lemma 1 and Daniell's theorem any positive linear form on $B$ extends into a positive measure on $E$, charging no polar sets. Therefore, it is null as soon as it is orthogonal to all continuous functions, and by the Hahn Banach theorem, any quasi-continuous function $g$ is limit in norm of a sequence of continuous functions $g_{n}$. A fortiori it is the limit in norm of the sequence $\tilde{g}_{n}, \tilde{g}_{n}$ being the truncation of $g_{n}$ by the upper and lower bounds of $g$. Taking a subsequence $n_{K}$ such that $\sum\left\|g-\tilde{g}_{n_{K}}\right\|<\infty$, one checks easily that $\tilde{g}_{n_{K}}$ converges towards $g$ quasi-uniformly.

REMARK. In the case of the deterministic translation process on the real line, quasi-continuous functions appear to be the continuous functions, as finely continuous functions are the right continuous functions.

\section{Various properties.}

3.1. We shall say that a function $f$ is quasi finely continuous iff it is finely continuous except on a polar set. An equivalent definition is that $f\left(X_{t}\right)$ is almost surely right continuous on $(0+\infty)$ and this property needs only to be checked $P_{m}$ a.s. (If $f$ is finely continuous except on a polar set $B$, and if $f\left(X_{t}\right)$ is not $P_{\mu}$ a.s. right continuous on $(0+\infty) P_{\mu}$ a.s. for some $\mu$, by the section theorem there is stopping time $T$ such that $f(X)_{T+} \neq f\left(X_{T}\right)$ and by the strong Markov property $X_{T} \in B P_{\mu}$ a.s., which yields a contradiction. Conversely, if $f$ is finely discontinuous on a nonpolar closed set $A$, by the strong Markov property, $f\left(X_{t}\right)$ is not $P_{m}$ a.s. right continuous at $T_{A} . T_{A}$ cannot be $P_{m}$ a.s. infinite, since $A$ is nonpolar, and therefore $m\left(e_{A}^{\alpha}\right)$ is positive.)

We shall say that a set is quasi finely open iff it is finely open except for a polar set, and quasi-open iff it is quasi finely open, and for any sequence $T_{n}$ announcing any predictable stopping time $T,\left\{X_{T} \in U\right\} \subseteq \lim \inf \left\{X_{T_{n}} \in U\right\}$. A quasi closed set will be the complementary of a quasi open set.

Proposition 2. A bounded function $f$ is quasi-continuous iff, for any open interval $(a b)$, the set $\{x, a<f(x)<b\}$ is quasi-open.

ProOF. The necessity is clear. To prove the sufficiency we may use reductio ad absurdum. Suppose a predictable time $T>0$ and an announcing sequence $T_{n}$ such that $\lim _{n} \sup \left|f\left(X_{T}\right)-f\left(X_{T_{n}}\right)\right| \geq \epsilon>0$ on a set $A$ of positive probability. (We fix an initial measure.) There is at least an interval $I$ of the form $(r-\epsilon / 3 r+\epsilon / 3)$, 
$r$ rational, such that the set $B=A \cap\left\{f\left(X_{T}\right) \in I\right\}$ has positive probability. Since $\{x, f(x) \in I\}$ is quasi-open, $f\left(X_{T_{n}}\right) \in I$ for $n$ large enough and $\omega \in B$. But then,

$$
\left|f\left(X_{T}\right)-f\left(X_{T_{n}}\right)\right|<2 \epsilon / 3
$$

and we get a contradiction.

COROLLARY. The following properties are equivalent:

(a) Every quasi finely continuous function is quasi-continuous.

(b) Every quasi finely open set is quasi-open.

(c) Every bounded $\alpha$-excessive function $f$ is regular, i.e. such that $f\left(X_{t}\right)$ is quasi left continuous.

ProOF. Clearly (a) follows from (b) by the proposition and (c) follows from (a). (b) follows from (c) by Theorem 4.5, p. 86 in [2]. In particular, this corollary applies to the case of classical potential theory and Brownian motion. It also applies to the case of Dirichlet spaces.

REMARK. With the argument given in Lemma 1, one easily checks that if the intersection of a decreasing family of quasi-closed sets is polar, they decrease quasi-uniformly to 0 .

3.2. PROPOSITION 3 (BALAYAge ON REgUlAR QUASI-ClOSED SETS). If $M$ is a regular and quasi-closed set and $f$ a quasi-continuous function, then $P_{T_{M}} f$ is quasi-continuous. In particular, $M$ is a projective set.

ProOF. One easily checks that the process $f\left(X_{T_{M}^{t}}\right)$ (with $T_{M}^{t}=T_{M} \circ \theta_{t}+t$ ) is quasi-continuous on $(0+\infty)$.

Its optional projection $P_{T_{M}} f\left(X_{t}\right)$ is therefore quasi-continuous (cf. step (iii) in the proof of the first equivalence).

3.3 Quasi-continuity and cofine continuity. In this section, we shall assume the existence of a Hunt process $\hat{X}_{t}$, with reference measure $m$, such that $U_{\alpha}$ and $\hat{U}_{\alpha}$ are in duality with respect to $m$. (Recall that $m$ is assumed to be bounded.)

THEOREM 2. A function is quasi-continuous iff it is quasi finely and quasi cofinely continuous.

ProOF. Let $\rho_{t}$ be the time reversal operator defined on $\{\omega, \zeta(\omega)>t\}$ by $X_{s}\left(\rho_{t} \omega\right)=X_{(t-s)-(\omega)}$ if $s<t$.

$$
X_{s}\left(\rho_{t} \omega\right)=\delta \quad \text { if } s \geq t .
$$

One checks easily on cylindric sets that, for any measurable set of paths $A$ in $\hat{\mathcal{F}}_{t}$

$$
E_{m}\left(\rho_{t}^{-1}(A) 1_{\{s>t\}}\right)=\hat{E}_{m}\left(A 1_{\{\zeta>t\}}\right) .
$$

Taking $A=\left\{\omega, f\left(X_{u}(\omega)\right)\right.$ is right continuous on $\left.(0 t)\right\}$ and letting $t$ take all positive values, it is clear that $f\left(\hat{X}_{u}\right)$ is $\hat{P}_{m}$ a.s. right continuous iff $f\left(X_{u-}\right)$ is $P_{m}$ a.s. left continuous.

\section{REFERENCES}

1. N. Aronszajn and K. Smith, Functional spaces and functional completion, Ann. Inst. Fourier (Grenoble) 6 (1955/1956), 125-185.

2. R. M. Blumenthal and R. K. Getoor, Markov processes and potential theory, Academic Press, New York, 1968. 
3. C. Dellacherie, Capacités et processus stochastiques, Springer-Verlag, Berlin and New York, 1972.

4. J. Deny, Théorie de la capacité dans les espaces fonctionnels, Séminaire Brelot-Choquet-Deny (1964/1965), Secrétariat Math., Paris, 1965.

5. __, Méthodes hilbertiennes en théorie du potentiel, (C.I.M.E. I Ciclo, Stresa, 1969), Edizioni Cremonese, Rome, 1970.

6. M. Fukushima, On the generation of Markov processes by symmetric forms, Proc. 2nd JapanUSSR Sympos. Probability Theory (Kyoto, 1972), Lecture Notes in Math., Vol. 330, Springer, Berlin and New York, 1973.

7. P. A. Meyer and J. Walsh, Quelques applications des résolvantes de Ray, Invent. Math. 14 (1971).

8. M. Silverstein, Symmetric Markov processes, Lecture Notes in Math., Vol. 426, Springer-Verlag, Berlin and New York, 1974.

Laboratoire de Probabilití P. et M. Curie, 75230 Paris Cedex 05, France

Current address: Laboratoire de Probalités, Université Paris VI, 4 Place Jussieu, 75230 Paris Cedex 05, France 